etc.). ${ }^{25}$ Es ist der Schutz der Integrität der Persönlichkeit, ${ }^{26}$ der durch eine Gewaltanwendung oder eine Drohung mit ihr verletzt wird, selbst wenn Körper und Gesundheit nicht beeinträchtigt sind. Oder anders ausgedrückt: Das Verhalten des Täters ist eine bewusste Missachtung der Persönlichkeit des Opfers und damit eine Überhebung der eigenen Persönlichkeit. ${ }^{27}$ Auf diese Weise lässt sich auch das Stalking als Form der Freiheitsverletzung verstehen. Und es lassen sich auch weitere Formen von Gewalt zivilrechtlich „bekämpfen", die nach der h. M. nicht vom Gewaltschutzgesetz umfasst wären. ${ }^{28}$ Beispiel ist die Drohung mit Gewalt gegen Sachen, die zu einer Freiheitsverletzung führt. Es kann z. B. ein Aufenthaltsverbot ausgesprochen werden, wenn von „U-BahnRowdys" mit dem Aufschlitzen einer Lederjacke, der Vernichtung des Schultascheninhalts oder mitgeführter Seminararbeiten gedroht wird, wenn der Betroffene weiterhin die Drohenden durch seine Anwesenheit „stört".

Dr. Dr. Herbert Grziwotz ist Notar in Regen, Lehrbeauftragter an der Universität Regensburg und an der FH Deggendorf sowie Gastdozent an der Bundesfinanzakademie.

\section{Fußnoten}

1S. nur Schumacher, WuM 2002, 420; vgl. auch Ehinger, FPR 1999, 262 ff. 2 Schumacher, FamRZ 2002, 645/646. 3 Vgl. hierzu $§ \S 1666,1666$ a BGB.

$4 \mathrm{Zu}$ den Anordnungen kurz Zwißler, GewaltschutzG, 2006, S. 19 ff. Zum Antrag s. Giers, FamRB 2005, 303 f. $5 \mathrm{~S}$. nur Schumacher/Janzen, Gewaltschutz in der Familie, 2003, Rn. 31.

6 RG, Urt. v. 12.1.1920 - VI 320/19, RGZ 97, 343/346; vgl. auch RG, Urt. v. 27.2.1904 - I 418/03, RGZ 58, 24/29, wonach besondere Persönlichkeitsrechte als sonstige Rechte nicht unter den Schutz des $\S 823$ Abs. 1 BGB fallen, u. RG, Urt. v. 11.4.1901 - VI 443/00, RGZ 48, 114/123, wonach nicht jede, die freie Willensbestimmung eines Anderen irgendwie beeinflussende Einwirkung unter den Begriff der Freiheitsverletzung fällt.

7 RG, Urt. v. 17.9.1908 - VI 423/07, in: Schubert/Glöckner, (Hrsg.), Nachschlagew. d. RG, BGB, Bd. 7, 1, 1999, § $823 \mathrm{~b}, 3$.

8 S. nur Endemann, Bürgerl. Recht, 8. Aufl. 1903, § 200 Fn. 28; Heller, Der privatrechtliche Schutz der Freiheit, 1917, S. 17 ff. u. Staudinger/Engelmann, BGB, 56. Aufl. 1910, § 823 Anm. II A 2 c.

9S. zu dieser Entwicklung nur Deutsch, in: FS f. Hauß, 1978, S. $43 / 44$ ff.; Wolf, in: FS f. von Hippel, 1967, S. 665/684; ders., JuS 1968, 77/79; Eckert, JuS $1994,625 / 627$ f. u. Leinemann, Der Begriff der Freiheit nach § 823 Abs. 1 BGB, 1969, S. 97 ff. Grundlegend war Oertmann, Verh. d. 28. DJT, Gutachten II, 1906, S. 33/58 f. Zum philosophischen Freiheitsbegriff s. Wolff, in:
FS f. Keller, 1989, S. 359/365 ff.

10 S. nur Palandt/Sprau, BGB, 65. Aufl. 2006, § 823 Rn. 6; weitergehend AnwKBGB/Katzenmeier, § 823 Rn. 5.

11 Offen OLG Köln, Urt. v. 6.2.2003 - 14 UF 249/02, FamRZ 2003, 1281; verneinend AnwK-BGB/Heinke, § 1 GewSchG Rn. 9 Fn. 26 (abw. Fn. 29); Palandt/Brudermüller, 65. Aufl. 2006, \$ 1 GewSchG Rn. 5 u. Weber, NJW 2004, 3084/3091.

12 Vgl. BT-Drs. 14/5429, S. 18 f. u. 28; s. dazu Schumacher/Janzen, a.a.O. (Fn. 6), Rn. 31 ff; AnwK-BGB/Heinke, $\S 1$ GewSchG Rn. 9; Palandt/Brudermüller, § 1 GewSchG Rn. 5 und im Ergebnis auch Ziegler, Das Gewaltschutzgesetz aus zivilrechtlicher Sicht, 2005, zugl. Diss. Mainz 2005, S. 48.

13 S. nur Palandt/Brudermüller, $\S 1$ GewSchG Rn. 5; Palandt/Sprau, § 823 Rn. 6; BGB-RGRK/Steffen, 12. Aufl. 1989, § 823 Rn. 14; Hk-BGB/Staudinger, 4. Aufl. 2005, § 823 Rn. 11; Soergel/Zeuner, BGB, 12. Aufl. 1998, § 823 Rn. 28; Staudinger/Hager, BGB, 13. Bearb. 1999, § 823 Rn. B 53 f. u Erman/Schiemann, BGB, 11. Aufl. 2004, § 823 Rn. 23; vgl. auch Schweikert/Baer, Das neue Gewaltschutzrecht, 2002, Rn. 271.

14 MünchKommBGB/Wagner, 4. Aufl. 2004, § 823 Rn. 92.

15 So insbes. MünchKommBGB/ Wagner, § 823 Rn. 92.

16 S. nur AnwK-BGB/Heinke, GewSchG § 1 Rn. 9; Ziegler, a.a.O. (Fn. 13), S. 48 u. Schumacher, FamRZ 2002, 645/648; zur entsprechenden Problematik bei § 823 Abs. 1 BGB s. Palandt/Thomas, § 823 Rn. 6 u. Staudinger/Hager, § 823 Rn. B 53.

17 S. nur Tröndle/Fischer, StGB, 52. Aufl.
2004, § 239 Rn. 2 u. Lackner/Kühl, StGB, 25. Aufl. 2004, § 239 Rn. 2.

18 Bejahend OLG Brandenburg, Beschl. v. 20.4.2005 - 9 UF 27/05, FamRB 2005, 330 u. AG Regensburg, Urt. v. 5.2.1999 - 9 C 2783/98, NJW-RR 1999, 1402; verneinend Jauernig/Teichmann, BGB, 11. Aufl. 2004, § 823 Rn. 5; unklar Bamberger/Roth/Spindler, § 823 Rn. 36 u. Rn. 39.

19 So bereits Soergel/Zeuner, $\S 823 \mathrm{Rn}$. 30.

20 Ebenso Soergel/Zeuner, § 823 Rn. 30.

21 Vgl. die Beispiele von Eckert, JuS 1994, $625 / 627$.

22 Umstr., s. nur Manssen, StaatsR II, 4. Aufl. 2005, Rn. 230 u. Dürig, in: Maunz/Dürig, GG, Art. 2 Abs. 2 Rn. 50 .

23 Murswiek, in: Sachs, GG, 3. Aufl. 2003, Art. 2 Rn. 230.

24 Offen bleibt, inwieweit generell die Entschließungsfreiheit unter den Schutz des $\S 823$ Abs. 1 BGB und des $\S$ 1 Abs. 1 S. 1 GewSchG fällt. Vgl. dazu die Nachw. Fußn. 10.

25 Vgl. auch Di Fabio, in: Maunz-Dürig, GG, Art. 2 Abs. 1 Rn. 59 u. Jarass, in: Jarass/Pieroth, GG, 7. Aufl. 2004, Art. 2 Rn. 6a. De lege ferenda ebenso Löhnig, Zivilrechtlicher Gewaltschutz, 2. Aufl. 2004, Rn. 86.

26 S. bereits Dürig, JR 1952, 259/261 u. ihm folgend Murswiek, in: Sachs, GG, Art. 2 Rn. 59.

27 Ähnlich Kaser, Röm. Privatrecht, 2. Aufl. 1971, S. 624 u. Pringsheim, ZSS. RA 52, 1932, 86 ff. zur römisch-rechtlichen actio iniuriarum.

28 Übersehen von AnwK-BGB/Heinke, § 1 GewSchG Rn. 28 Fn. 28.

\title{
Wie wird die Kriminalpolitik 2006/2007 aussehen?
}

\section{Monika Frommel}

Auf die Frage, ob unter der neuen Bundesregierung „härter bestraft “ würde, antwortete die alte und zugleich neue Justizministerin kurz nach der Bildung der neuen Regierung, ein Paradigmenwechsel erfolge nicht (TAZ vom 15.11.2005). Die Frage reagierte auf ein nur noch in Randbereichen zutreffendes Stereotyp, wonach Konservative tendenziell für einen Abbau von Bürgerrechten und für härtere Strafen votierten, während Grüne und Liberale sich eher als Aufklärer und Vertreter einer alternativen Kriminalpolitik sehen möchten. Rot-schwarz müsste nach dieser Einordnung somit eher als rot-grün für eine punitive Linie eintreten. Schaut man sich aber die Kriminalpolitik der letzten Jahre und die Entwürfe für diese Legislaturperiode an, dann fällt es zunehmend schwer, diese Frontlinien noch zu identifizieren. In den letzten Jahren waren alle strafrechtlichen Neuregelungen Kompromisse, der Sache nach also schon lange das Ergebnis einer großen Koalition in den Ausschüssen. Unterschiede erkennt man also nur in einem Bereich, dem klassischen Sicherheitsstrafrecht. Dort fällt auf, dass die CDU/CSU auf der einen und die Grünen auf der anderen, wohl auch die neue Linkspartei, unterschiedliche Anforderungen an die Feststellung dessen, was man gemeinhin so als „gefährlich“ versteht, stellen, wobei es bisweilen zu einer mehr als brüchigen ge- meinsamen Haltung der Opposition, also zusammen mit der FDP gibt. Der Politikstil der letzten Jahre war eine sektorale Strafausweitung (Ostendorf, Bewährungshilfe 2005, 59) und die Träger dieser Forderungen waren durchaus auch soziale Bewegungen. Eine verbesserte und ggf. auch klügere strafrechtliche Kontrolle von häuslicher Gewalt etwa ist eine typische Forderung der europäischen Frauenbewegung der letzten Jahrzehnte. Diese hat sich zwar inhaltlich verfeinert. Selbstverständlich befördert diese soziale Bewegung auch das sog. Sicherheitsstrafrecht, aber nicht wegen autoritärer, sondern wegen eines Opferschutz-Konzeptes, das leider nicht sehr professionelle ist. Immerhin waren die Kernforderungen auf eine Reform des Zivilrechts gerichtet. Eigentlich müssten sie dafür eintreten, in dieser Legislaturperiode die Reform des FGG-Verfahrens voran zu treiben, um Stalking und Gewalt in allen Lebensbereichen flexibel unterbinden zu können. Es kennzeichnet nun einmal eine egalitäre Gesellschaft neue und ggf. für einige wenige auch härtere Kontrollstile zu entwickeln und keine Realpolitik kann sich auf Dauer den Forderungen nach flexiblem Opferschutz widersetzen, zumal dieses Thema medial gut ankommt, medial stark vermarktet wird und die von Juristen geäußerten Bedenken wenig überzeugen. Sicherheit ist eben auch ein Bürgerrecht, und die Instrumentalisierung von Bürgerrechten für eine dumme Sicherheitspolitik bzw. für eine Placebo-Politik ist eben schwer zu erkennen. 